\title{
Restless leg syndrome in hospitalized psychiatric patients in Lebanon: a pilot study
}

\author{
This article was published in the following Dove Press journal: \\ Neuropsychiatric Disease and Treatment \\ 11 October 2016 \\ Number of times this article has been viewed
}

\author{
Farid Talih' \\ Jean Ajaltouni' \\ Firas Kobeissy² \\ 'Department of Psychiatry, \\ ${ }^{2}$ Department of Biochemistry and \\ Molecular Genetics, Faculty of \\ Medicine, American University of \\ Beirut, Beirut, Lebanon
}

\begin{abstract}
Objectives: To characterize and describe the prevalence of restless leg syndrome (RLS) in hospitalized psychiatric patients and to investigate the correlations between patient profile and RLS.

Methods: Demographic information, psychiatric diagnoses, psychotropic medication use, and history of substance use were collected from hospitalized psychiatric patients at the American University of Beirut Medical Center; Beirut, Lebanon. A validated questionnaire to evaluate RLS symptomatology was also administered to 126 participants who agreed to participate, as well as questionnaires for insomnia, depression, and anxiety symptoms. Statistical analysis was conducted to detect the prevalence of RLS among the participants and to examine correlations with RLS in a hospitalized psychiatric population.

Results: Out of the 126 participants who completed the survey, RLS was detected in $18 \%$ of the participants. Of interest, RLS was also found to be associated with higher depressive symptomatology, suicidal ideation, and working night shifts.
\end{abstract}

Keywords: restless leg syndrome, insomnia, depression, and anxiety symptoms

\section{Introduction}

Sleep is an important part of overall health, influencing both physical and mental health. ${ }^{1}$ Psychiatric disorders are common conditions that cause significant morbidity and impairment. ${ }^{2}$ Sleep disturbances are also common and widespread; they also tend to be persistent and chronic. ${ }^{3}$ Sleep disorders can have various etiologies and multiple sleep disorders can co-occur in the same individual. The close and overlapping relationship between many psychiatric and sleep disorders has been established for some time now in the literature. ${ }^{4,5}$ According to the Diagnostic and Statistical Manual of Mental Disorders, fifth edition, diagnostic criteria for psychiatric disorders include sleep disturbances as a major criterion in the diagnosis of many psychiatric conditions, including depression, mania, psychotic disorders, and posttraumatic stress disorder., ${ }^{6,7}$

Psychiatric medications can have a positive modulating effect on sleep, sometimes enhancing sleep architecture ${ }^{8}$ however, psychotropic medications can have negative effects on sleep patterns. Some antipsychotics can improve nocturnal sleep, but cause daytime somnolence. ${ }^{8}$ Selective serotonin reuptake inhibitors can improve daytime alertness but may exacerbate restless legs syndrome (RLS) at night, causing reduced sleep quality. ${ }^{8}$ RLS is a sleep-related movement disorder characterized by an urge to move the extremities, usually the legs; it has been found to be more prevalent in females and the elderly. ${ }^{9}$ The clinical and diagnostic features of RLS as per the international classification of sleep disorders, third edition, include an urge to move the legs accompanied by pain or discomfort, exacerbated in the evening and at rest, 
which is usually relieved by moving the extremities. ${ }^{10}$ Factors associated with RLS include iron deficiency, dopamine antagonists, antidepressants, pregnancy, chronic renal failure, and prolonged immobility. ${ }^{11}$ Nicotine and caffeine have been implicated in RLS but the link remains unclear. ${ }^{12,13}$

The pathophysiology of RLS has been associated with iron deficiency. Iron is an essential component in brain dopamine synthesis; altered dopaminergic pathways along with iron deficiency have been found to play a role in the development of RLS. ${ }^{14}$ The pathophysiology of primary RLS is postulated to be a combination of genetic predisposition with iron deficiency causing an altered dopaminergic pathway. RLS may lead to a disrupted sleeping pattern, mood and cognitive disorders, cardiovascular morbidity, and reduced quality of life. ${ }^{15}$ RLS, in addition to causing difficulty in falling asleep (mimicking insomnia), is associated with fragmentation of sleep and frequent sleep arousals. ${ }^{16}$ It is most likely that the disruption of sleep in RLS is due to the urge to move the legs or actual mechanical movement of the legs. Additionally, the use of medications to alleviate leg discomfort can potentially exacerbate psychiatric problems, such as addiction to sedating agents. ${ }^{17}$ Medications such as benzodiazepines and opiate-based sedatives used by patients to alleviate nocturnal leg discomfort can exacerbate sleep problems by causing daytime hypersomnolence. ${ }^{18}$ Certain dopamine agonists used to alleviate leg discomfort during sleep can exacerbate impulsive behaviors such as gambling. ${ }^{19}$

This pilot study aims to determine the prevalence of RLS in a sample of hospitalized psychiatric patients. We hypothesize that there will be a high prevalence of RLS in our sample, which will be higher than the prevalence in the general population. In Lebanon, there are sparse data on sleep disorders in general (mostly limited to insomnia) and none, to the best of our knowledge, on RLS, with no recent updates on its prevalence and characterization. ${ }^{20,21} \mathrm{We}$ will also examine associations and putative correlations among RLS and different psychiatric disorders and associated findings in Lebanese psychiatric patients with RLS.

\section{Methods}

A cross-sectional survey-based questionnaire was developed to investigate sleep disorders among hospitalized psychiatric patients. Research assistants trained in administering sleep questionnaires by the principal investigator, a psychiatrist specializing in sleep medicine, conducted the surveys. The study was conducted at the American University of Beirut Medical Center (AUBMC), Beirut, Lebanon, and was approved by the American University of Beirut Institutional
Review Board. The native language is Arabic and the questionnaires used in this study are validated in Arabic.

The participants were individuals hospitalized at the inpatient psychiatry unit at AUBMC and were recruited by the investigators after obtaining written informed consent. Over a period of 2 years, all eligible hospitalized psychiatric patients were approached to participate in this study and 126 patients consented to participate. This facility admits around 300 patients per year. Consent was obtained after the research goals, risks, and benefits were explained to the potential participants. This was a self-report-based survey and the participants were assisted, as needed, by the research assistants if they requested assistance in clarifying or explaining a survey item. Exclusion criteria included being $<18$ years of age and suffering from cognitive impairment that would impede the participant from interacting with the investigators such as dementia or profound developmental disorders. All patients presenting to the psychiatry unit were asked to participate. The survey included demographic and medical information, psychiatric diagnoses (as per the admitting psychiatrist who based the diagnoses on the Diagnostic and Statistical Manual, fifth edition criteria), working night shifts, use of substances (alcohol, drugs, caffeine, and nicotine), and current medications. Working night shifts was assessed via a single "yes or no" item in the survey. For our purposes, working night shift was defined as working throughout the night until the early morning. The patient health questionnaire (PHQ-9) and the generalized anxiety disorder-7 (GAD-7) questionnaire were used to evaluate depression and anxiety symptomatology. The PHQ-9 questionnaire assesses depressive symptomatology.22 For each item, participants indicated whether, during the last 2 weeks, the symptom had bothered them "not at all," "several days," "more than half the days," or "nearly every day." Each item yields a score of 0 to 3 and scores range from 0 to 27 ; scores of 10-14, 15-19, and 20 or greater correspond to moderate, moderately severe, and severe depression, respectively. A score of 10 or greater has a sensitivity of $93 \%$ and a specificity of $88 \%$ for the detection of depression. ${ }^{22}$

The GAD-7 scale, a 7-item scale, is a screening tool for anxiety used in clinical practice and research. ${ }^{23}$ For each item, the participants indicated whether during the past 2 weeks the symptom had bothered them as "not at all," "several days," "more than half the days," or "nearly every day." The total was calculated by assigning scores of $0,1,2$, and 3 to the above categories, respectively. Scores of 5, 10, and 15 were taken as the cutoff points for mild, moderate, and severe anxiety. Using the threshold of 10 , it has a sensitivity of 
$89 \%$ and a specificity of $82 \%$ for detecting anxiety. ${ }^{23}$ Both the PHQ-9 and the GAD-7 are validated tools used to assess symptoms of depression and anxiety, respectively. They have also been found to be reliable and validated in Arabic. ${ }^{24,25}$ The RLS rating scale is a validated scale that measures RLS symptomatology using a ten-item questionnaire to assess the severity of RLS symptoms as mild, moderate, and severe. ${ }^{26}$ The questionnaire consists of ten items addressing the severity of RLS, its effect on mood, sleep discomfort, and daily affairs. The items are rated from 0 to $4 ; 0$ being no discomfort and 4 being very severe discomfort. Mild RLS corresponds to a score from 1-10, moderate RLS corresponds to a score from 11-20, severe RLS from 21-30, and very severe RLS from 31-40. The RLS rating scale has been validated in Arabic and found to be a reliable tool in measuring RLS in Arabic-speaking populations. ${ }^{27}$

Collected data was entered into a database and was subsequently statistically analyzed using SPSS ${ }^{\circledR}$ (IBM Corporation, Armonk, NY, USA) for Windows ${ }^{\circledR}$ version 20 (Microsoft Corporation, Redmond, WA, USA). We first examined the prevalence of RLS. Any demographic factors that might correlate with this disorder were analyzed. We also attempted to detect correlations among psychiatric conditions and RLS. Bivariate analysis was performed to examine correlations between patient demographics and characteristics, RLS, the primary psychiatric diagnoses, and any substance use. Associations between RLS and the type of psychiatric diagnoses were assessed using Pearson's chi-square test. All tests were two-sided with a type one error of 0.05 . The RLS-related findings presented here are preliminary results as part of a larger pilot study looking at the prevalence of multiple sleep disorder among hospitalized psychiatric patients.

\section{Results}

Among the 126 participants who completed the survey $44 \%$ were diagnosed with depression, $12 \%$ with schizophrenia or a psychotic disorder, $18 \%$ with bipolar disorder, and $16 \%$ with substance use disorders (Table 1). Additionally, 34\% reported taking over-the-counter sleeping aids, $11 \%$ reported using cannabis, $2 \%$ reported using illicit opiates or heroin, and $7 \%$ were using nonprescribed benzodiazepines.

Screening with the RLS rating scale in our sample showed that $18 \%$ of the participants were suffering from restless legs symptoms and 50\% of those had moderateto-severe RLS symptomatology. RLS was correlated with depressive symptoms $(r=0.202, P<0.05)$ and working night shifts $(r=0.202, P<0.05)$. Higher PHQ-9 scores (more depressive symptomatology) were associated with a higher
Table I Baseline characteristics

\begin{tabular}{|c|c|c|}
\hline Variable & $\mathbf{N}(\%)$ & Mean RLS score \\
\hline \multicolumn{3}{|l|}{ Sex } \\
\hline Male & $54(43)$ & I.44 $(P=0.44)$ \\
\hline Female & $72(57)$ & 2.44 \\
\hline \multicolumn{3}{|l|}{ Age (years) } \\
\hline $18-25$ & 37 & $4.66(P=0.59)$ \\
\hline $26-35$ & 26 & 2.71 \\
\hline $36-45$ & 32 & 1.54 \\
\hline $46-55$ & 17 & 1.38 \\
\hline$>55$ & 14 & 0.00 \\
\hline \multicolumn{3}{|l|}{ Marital status } \\
\hline Single & $63(50)$ & I.88 $(P=0.33)$ \\
\hline Married & $50(35)$ & 1.55 \\
\hline Divorced or widowed & $12(14)$ & 4.09 \\
\hline Widowed (add to above) & $I(<I)$ & $\mathrm{n} / \mathrm{a}$ \\
\hline \multicolumn{3}{|l|}{ Sleep hours } \\
\hline$<7$ hours & $62(50.5)$ & $4.7(P=0.11)$ \\
\hline $7-8$ hours & $32(25.4)$ & 0.30 \\
\hline$>8$ hours & $32(25.4)$ & 0.44 \\
\hline \multicolumn{3}{|l|}{ Diagnosis } \\
\hline Depression & $56(44)$ & $4.27(P=0.44)$ \\
\hline Schizophrenia/schizoaffective & $17(13.5)$ & 0.42 \\
\hline \multicolumn{3}{|l|}{ disorder } \\
\hline Bipolar & $23(18)$ & 2.34 \\
\hline Anxiety disorder & $10(9)$ & 2.22 \\
\hline Substance use disorder & $19(15.5)$ & 2.21 \\
\hline Eating disorder & $\mathrm{I}(\mathrm{I})$ & 0 \\
\hline \multicolumn{3}{|l|}{ Depressive symptoms } \\
\hline None-to-mild & $13(10.37)$ & $0(P=0.02)$ \\
\hline Moderate-to-severe & $113(89.63)$ & 2.68 \\
\hline \multicolumn{3}{|l|}{ Anxiety symptoms } \\
\hline None-to-mild & $42(33.3)$ & $1.55(P=0.14)$ \\
\hline Moderate-to-severe & $84(70.0)$ & 4.63 \\
\hline
\end{tabular}

Abbreviations: RLS, restless leg syndrome; $n / a$, not applicable.

likelihood of RLS (Table 2). Among participants with severe depressive symptomatology (per PHQ-9); 34\% were found to have RLS as per Table 2. Among those with suicidal ideation (per item 9 on PHQ-9); $21 \%$ were found to have RLS $\left(\chi^{2}=6.38, P=0.01\right)$.

Sex, age, and psychiatric diagnoses did not significantly correlate with RLS as shown in Table 1. Regarding nicotine and caffeine use, there was no correlation between the use of caffeine and/or nicotine and RLS symptomatology (Table 3).

Table 2 Depressive symptomatology and RLS

\begin{tabular}{|c|c|c|c|}
\hline Depressive symptoms $^{a}$ & $\frac{\text { No RLS }}{\mathbf{N}(\%)}$ & $\frac{\text { RLS }}{\text { N (\%) }}$ & $\begin{array}{l}\text { Chi-square } \\
\text { test }\end{array}$ \\
\hline None-to-mild & $13(100)$ & $0(0)$ & $\chi^{2}=10.1, P=0.01$ \\
\hline $\begin{array}{l}\text { Moderate to moderately } \\
\text { severe }\end{array}$ & $50(94)$ & $3(6)$ & \\
\hline Severe & $40(66)$ & $20(34)$ & \\
\hline
\end{tabular}

Note: ${ }^{2}$ As per PHQ-9 score.

Abbreviations: PHQ-9, patient health questionnaire; RLS, restless leg syndrome. 
Table 3 Prevalence of caffeine and nicotine use in patients with RLS

\begin{tabular}{lllll}
\hline RLS & $\begin{array}{l}\text { Frequency } \\
(\mathbf{n = 1 2 6 )}\end{array}$ & Percentage (\%) & $\begin{array}{l}\text { Nicotine } \\
\text { use (\%) }\end{array}$ & $\begin{array}{l}\text { Caffeine } \\
\text { use (\%) }\end{array}$ \\
\hline No & 103 & 81 & 87.5 & 84 \\
Mild & 11 & 9 & 3 & 6.7 \\
Moderate & 10 & 8 & 6.3 & 6.7 \\
Severe & 2 & 1 & 3.2 & 2.2 \\
\hline
\end{tabular}

Abbreviation: RLS, restless leg syndrome.

\section{Discussion}

To the best of our knowledge, this pilot study is the first of its kind to examine the prevalence of RLS in hospitalized psychiatric patients in Lebanon and the Middle East region. Our findings suggest that the presence of a sleep disorder such as RLS can be a detrimental factor for the mental health of psychiatric patients. In our sample, it is reasonable to assume that participants were suffering from severe psychiatric illness as they were a hospitalized population and the additional burden of sleep disorders was potentially a factor that could be mitigated if recognized and treated. This is of particular importance in Lebanon, a country with high levels of psychosocial stress that has suffered from several decades of armed conflict, ongoing violence, and political instability. These factors have been shown to significantly increase the odds ratio of developing mental health disorders among the Lebanese; as well as increasing the probability of traumatic brain injuries and other injuries that can exacerbate pre-existing psychiatric disorders. ${ }^{28,29}$

The overall prevalence of RLS in Lebanon and the neighboring Arab region is unknown. A Saudi study found that renal patients with insomnia had a high prevalence of RLS at $62 \%{ }^{30}$ The overall prevalence of RLS has been estimated at 5\%-10\% in European and North American populationbased studies. The prevalence of clinically significant RLS is $2 \%-3 \%$ in Caucasians, but is lower in Asians, and rates among Asians have been reported as $0.8 \%-2.2 \% .^{31,32}$ The prevalence of restless legs symptoms in our study was $18 \%$, with $50 \%$ of those screening positive for RLS having moderate-to-severe symptoms. Females and older adults have been reported to have a higher prevalence of RLS; 9 this was not detected in our sample, possibly due to our sample size. The detected prevalence of clinically significant RLS is on the high side when compared to the available literature and this is of interest (and concern) as we did detect a significant association between suicidal ideation and RLS in our population.

As an altered dopaminergic state is the core pathophysiology behind RLS, the association of RLS severity with suicidal ideation may be reflective of a hypodopaminergic state, which also exacerbates depression. Alternatively, those who have suicidal ideation (sicker patients) may be taking more psychotropic medications that potentially exacerbate RLS. Recent studies have supported associations between depression and RLS, ${ }^{33}$ suicidality has also been associated with insomnia severity. ${ }^{34}$ Nicotine and caffeine have an unclear association with RLS: they were thought to worsen RLS; however, recent population studies did not find a significant link with RLS. ${ }^{12,13}$ Our findings regarding caffeine, nicotine, and RLS are consistent with the recent literature.

The significant association between working in the night shift and RLS raises the possibility that those with difficulty sleeping at night due to RLS symptoms and secondary insomnia may choose to work in night shifts more than their peers.

\section{Limitations}

This study has several limitations. The relatively small sample size is a significant limitation. The psychiatric unit at AUBMC cares for a diverse clinical patient population and annually admits $\sim 300$ patients, yet it was challenging to recruit patients as many of them were not psychiatrically stable or cognitively intact to participate. We plan to use the data obtained from this pilot study for a larger scale study. Additionally, this is a pilot study that aims to develop lines of inquiry into sleep disorders in Lebanon. Another limitation is that psychotropic medication effects were not controlled for in our study; however, this was not feasible given the diverse and heterogeneous patient population hospitalized at our unit and the small population size. However, assuming that antipsychotics, which are primarily dopamine antagonists, would exacerbate RLS, we did not find that psychotic patients (more likely to be taking antipsychotics) differed from other psychiatric patients in the prevalence of RLS. Generalizability may be a concern, as this was a Lebanese population with relatively severe psychiatric illness (hospitalized); however, we feel this is mitigated by the fact that clinically it was a diverse clinical psychiatric population and the results highlight the importance of addressing sleep-related issues, especially RLS among psychiatric patients.

A future goal of a larger follow-up study is to collect serum and plasma for biochemical analysis for possible markers that can be related to RLS, which are briefly discussed. Our findings are consistent with the growing evidence in the medical literature that suggests underlying links between psychiatric and sleep disorders. It appears to be a complex bidirectional relationship that suggests an underlying molecular basis for coexisting sleep and psychiatric disorders. Iron, 
ferritin, and dopaminergic activity, for example, are common underlying links between RLS and some psychiatric disorders. ${ }^{14}$ Up to $90 \%$ of depressed patients have reported sleep disturbances as a symptom of their depression. ${ }^{35,36}$

Sleep-related symptoms may serve as biomarkers for mood disorders. For example, major depression has been associated with REM sleep abnormalities. ${ }^{37-39}$ Previous studies on RLS suggested that decreased slow wave sleep and increased REM density were predictive factors for depressive symptomatology. ${ }^{40}$ Increased REM sleep latency and decreased slow wave sleep were found to be present in patients with RLS when compared to patients with major depressive disorders without RLS. ${ }^{41}$ RLS has been found to be associated with a reduced locus of cognitive emotional control, negative personality traits, and a poor quality of life. ${ }^{42}$ Biomarkers such as plasminogen activator inhibitor type 1 and interleukin 6 were also found to be associated with both mood and sleep disorders. Plasminogen activator inhibitor type 1 is involved in circadian clock regulation, and was found to be a potential link between depression and comorbid sleep disorders. ${ }^{43,44}$ Nonspecific indicators, such as interleukin 6 , associated with depression have been detected to be elevated in sleep disorders. ${ }^{45}$ Bipolar disorder and delayed sleep phase syndrome appear to have common genetic basis. Research does seem to show a molecular basis connecting psychiatric and sleep disorders. ${ }^{46}$ Additionally, sleep disorders were found to be highly prevalent in individuals hospitalized for substance abuse disorders. ${ }^{47}$

\section{Conclusion}

Given the higher than average prevalence of RLS detected in our study, it is important to systematically screen hospitalized psychiatric patients for RLS and address RLS as a specific entity within the overall context of the primary mental illness. Collaboration with a sleep specialist is recommended in complex cases with a challenging sleep presentation. Improving sleep and addressing sleep disorders will have an overall positive effect on the mental health of psychiatric patients.

\section{Acknowledgment}

Authors are very thankful for the staff of the inpatient psychiatry unit at AUBMC for facilitating this work and assisting the investigators in data collection.

\section{Author contributions}

FT, JA, and FK worked on study conception and design. FT and JA analyzed and interpreted the data. FT, JA, and FK critically revised the manuscript with input from the entire team. All authors have read and approved the final draft.

\section{Disclosure}

The authors report no conflicts of interest in this work.

\section{References}

1. Cinosi E, Di Iorio G, Acciavatti T, et al. Sleep disturbances in eating disorders: a review. Clin Ter. 2011;162(6):e195-e202.

2. Abe Y, Suganuma T, Ishii M, et al. Association of genetic, psychological and behavioral factors with sleep bruxism in a Japanese population. J Sleep Res. 2012;21(3):289-296.

3. Stranges S, Tigbe W, Gómez-Olivé FX, Thorogood M, Kandala N-B. Sleep problems: an emerging global epidemic? Findings from the INDEPTH WHO-SAGE study among more than 40,000 older adults from 8 countries across Africa and Asia. Sleep. 2012;35(8):1173-1181.

4. Sateia MJ. Update on sleep and psychiatric disorders. Chest J. 2009; 135(5):1370-1379.

5. Etain B, Dumaine A, Bellivier F, et al. Genetic and functional abnormalities of the melatonin biosynthesis pathway in patients with bipolar disorder. Hum Mol Genet. 2012;21(18):4030-4037.

6. Batterham PJ, Glozier N, Christensen H. Sleep disturbance, personality and the onset of depression and anxiety: prospective cohort study. Aust N Z J Psychiatry. 2012;46(11):1089-1098.

7. Sivertsen B, Posserud M-B, Gillberg C, Lundervold AJ, Hysing M. Sleep problems in children with autism spectrum problems: a longitudinal population-based study. Autism. 2012;16(2):139-150.

8. Waters F, Faulkner D, Naik N, Rock D. Effects of polypharmacy on sleep in psychiatric inpatients. Schizophr Res. 2012;139(1):225-228.

9. Chahine LM, Chemali ZN. Restless legs syndrome: a review. CNS Spectr. 2006;11(07):511-520.

10. Allen RP, Picchietti DL, Garcia-Borreguero D, et al. Restless legs syndrome/Willis-Ekbom disease diagnostic criteria: updated International Restless Legs Syndrome Study Group (IRLSSG) consensus criteria-history, rationale, description, and significance. Sleep Med. 2014; 15(8):860-873.

11. Allen RP, Picchietti D, Hening WA, Trenkwalder C, Walters AS, Montplaisi J. Restless legs syndrome: diagnostic criteria, special considerations, and epidemiology: a report from the restless legs syndrome diagnosis and epidemiology workshop at the National Institutes of Health. Sleep Med. 2003;4(2):101-119.

12. Bayard M, Avonda T, Wadzinski J. Restless legs syndrome. Am Fam Physician. 2008;78(2):235-240.

13. Montplaisir J. Cigarette smoking as a risk factor or an exacerbating factor 4 for restless legs syndrome and sleep bruxism. Sleep. 1997; 20(4):290-293.

14. Dauvilliers Y, Winkelmann J. Restless legs syndrome: update on pathogenesis. Curr Opin Pulm Med. 2013;19(6):594-600.

15. Trenkwalder C, Paulus W. Restless legs syndrome: pathophysiology, clinical presentation and management. Nat Rev Neurol. 2010;6(6): 337-346.

16. Picchietti DL, Wang VC, Picchietti MA. Intravenous iron given prior to pregnancy for restless legs syndrome is associated with remission of symptoms. J Clin Sleep Med. 2012;8(5):585.

17. Ghandour LA, El Sayed DS, Martins SS. Prevalence and patterns of commonly abused psychoactive prescription drugs in a sample of university students from Lebanon: an opportunity for cross-cultural comparisons. Drug Alcohol Depend. 2012;121(1):110-117.

18. Rinaldi F, Galbiati A, Marelli S, Strambi LF, Zucconi M. Treatment options in intractable restless legs syndrome/Willis-Ekbom disease (RLS/WED). Curr Treat Options Neurol. 2016;18(2):1-9.

19. Engeln M, Ansquer S, Dugast E, Bezard E, Belin D, Fernagut P-O. Multi-faceted impulsivity following nigral degeneration and dopamine replacement therapy. Neuropharmacology. 2016;109:69-77. 
20. Choueiry N, Salamoun T, Jabbour H, El Osta N, Hajj A, Khabbaz LR. Insomnia and relationship with anxiety in university students: a crosssectional designed study. PloS one. 2016;11(2):e0149643.

21. Assaad S, Costanian C, Haddad G, Tannous F. Sleep patterns and disorders among university students in Lebanon. J Res Health Sci. 2014; 14(3):198-204.

22. Spitzer RL, Kroenke K, Williams JB. Group PHQPCS. Validation and utility of a self-report version of PRIME-MD: the PHQ primary care study. JAMA. 1999;282(18):1737-1744.

23. Spitzer RL, Kroenke K, Williams JB, Löwe B. A brief measure for assessing generalized anxiety disorder: the GAD-7. Arch Intern Med. 2006;166(10):1092-1097.

24. Gilbody S, Richards D, Brealey S, Hewitt C. Screening for depression in medical settings with the patient health questionnaire (PHQ): a diagnostic meta-analysis. J Gen Intern Med. 2007;22(11):1596-1602.

25. Sawaya H, Atoui M, Hamadeh A, Zeinoun P, Nahas Z. Adaptation and initial validation of the patient health questionnaire-9 (PHQ-9) and the generalized anxiety disorder-7 questionnaire (GAD-7) in an Arabic speaking Lebanese psychiatric outpatient sample. Psychiatry Res. 2016;239:245-252.

26. Group IRLSS. Validation of the International Restless Legs Syndrome Study Group rating scale for restless legs syndrome. Sleep Med. 2003; 4(2):121-132.

27. Ahmed AE. Validation of Arabic versions of three sleep surveys. Qatar Med J. 2014;2014(2):130.

28. Karam EG, Mneimneh ZN, Karam AN, et al. Prevalence and treatment of mental disorders in Lebanon: a national epidemiological survey. The Lancet. 2006;367(9515):1000-1006.

29. Karam G, Itani L, Fayyad J, Karam A, Mneimneh Z, Karam E. Prevalence, correlates, and treatment of mental disorders among Lebanese older adults: a national study. Am J Geriatr Psychiatry. 2016;24(4):278-286.

30. Al-Jahdali HH, Al-Qadhi WA, Khogeer HA, Al-Hejaili FF, Al-Ghamdi SM, Al Sayyari AA. Restless legs syndrome in patients on dialysis. Saudi J Kidney Dis Transpl. 2009;20(3):378.

31. Ohayon MM, O'Hara R, Vitiello MV. Epidemiology of restless legs syndrome: a synthesis of the literature. Sleep Med Rev. 2012;16(4): 283-295.

32. Allen RP, Stillman P, Myers AJ. Physician-diagnosed restless legs syndrome in a large sample of primary medical care patients in western Europe: prevalence and characteristics. Sleep Med. 2010;11(1):31-37.

33. Lee HB, Song ML, Koo BB, Cho YW. Mood symptoms and restless legs syndrome without periodic limb movements during sleep: is it a clinical subtype? J Neuropsychiatry Clin Neurosci. Epub 2016 Jul 15.

34. McCall WV, Blocker JN, D'Agostino R, et al. Insomnia severity is an indicator of suicidal ideation during a depression clinical trial. Sleep Med. 2010;11(9):822-827.
35. Liu Y, Croft JB, Wheaton AG, et al. Association between perceived insufficient sleep, frequent mental distress, obesity and chronic diseases among US adults, 2009 behavioral risk factor surveillance system. BMC Public Health. 2013;13(1):1.

36. Dagan Y, Stein D, Steinbock M, Yovel I, Hallis D. Frequency of delayed sleep phase syndrome among hospitalized adolescent psychiatric patients. J Psychosom Res. 1998;45(1):15-20.

37. Pillai V, Kalmbach DA, Ciesla JA. A meta-analysis of electroencephalographic sleep in depression: evidence for genetic biomarkers. Biol Psychiatry. 2011;70(10):912-919.

38. Kupfer D. Application of EEG sleep for the differential diagnosis and treatment of affective disorders. Pharmakopsychiatr Neuropsychopharmakol. 1978;11(1):17-26.

39. Palagini L, Baglioni C, Ciapparelli A, Gemignani A, Riemann D. REM sleep dysregulation in depression: state of the art. Sleep Med Rev. 2013; 17(5):377-390.

40. Brand S, Beck J, Hatzinger M, Savic M, Holsboer-Trachsler E. Unfavorable polysomnographic sleep patterns predict poor sleep and poor psychological functioning 3 years later in patients with restless legs syndrome. Neuropsychobiology. 2010;63(2):92-102.

41. Brand S, Lehtinen A, Hatzinger M, Holsboer-Trachsler E. Comparison of sleep EEG profiles of patients suffering from restless legs syndrome, restless legs syndrome and depressive symptoms, and major depressive disorders. Neuropsychobiology. 2009;61(1):41-48.

42. Brand S, Beck J, Hatzinger M, Holsboer-Trachsler E. Patients suffering from restless legs syndrome have low internal locus of control and poor psychological functioning compared to healthy controls. Neuropsychobiology. 2013;68(1):51-58.

43. Gottesmann C, Gottesman I. The neurobiological characteristics of rapid eye movement (REM) sleep are candidate endophenotypes of depression, schizophrenia, mental retardation and dementia. Prog Neurobiol. 2007;81(4):237-250

44. Mou X, Peterson CB, Prosser RA. Tissue-type plasminogen activatorplasmin-BDNF modulate glutamate-induced phase-shifts of the mouse suprachiasmatic circadian clock in vitro. Eur J Neurosci. 2009;30(8):1451-1460.

45. Prather AA, Rabinovitz M, Pollock BG, Lotrich FE. Cytokine-induced depression during IFN- $\alpha$ treatment: the role of IL- 6 and sleep quality. Brain Behav Immun. 2009;23(8):1109-1116.

46. Miller AH, Maletic V, Raison CL. Inflammation and its discontents: the role of cytokines in the pathophysiology of major depression. Biol Psychiatry. 2009;65(9):732-741.

47. Mahfoud Y, Talih F, Streem D, Budur K. Sleep disorders in substance abusers: how common are they? Psychiatry (Edgmont). 2009; 6(9):38-42.
Neuropsychiatric Disease and Treatment

\section{Publish your work in this journal}

Neuropsychiatric Disease and Treatment is an international, peerreviewed journal of clinical therapeutics and pharmacology focusing on concise rapid reporting of clinical or pre-clinical studies on a range of neuropsychiatric and neurological disorders. This journal is indexed on PubMed Central, the 'PsycINFO' database and CAS,

\section{Dovepress}

and is the official journal of The International Neuropsychiatric Association (INA). The manuscript management system is completely online and includes a very quick and fair peer-review system, which is all easy to use. Visit http://www.dovepress.com/testimonials.php to read real quotes from published authors. 